\title{
A simple phenotyping method for deep-rooting rice grown in pots
}

\author{
Jae-Hyuk Han $\cdot$ Na-Hyun Shin $\cdot$ Jae-Hoon Moon $\cdot J o o n g$ Hyoun Chin $\cdot$ Soo-Cheul Yoo
}

Received: 6 December 2016 / Revised: 15 December 2016 / Accepted: 26 December 2016

(c) Korean Society for Plant Biotechnology

\begin{abstract}
Deep rooting, which enables plants to extract water from greater soil depths, is a critical strategy for improving plant survival under water-deficient conditions. However, as it is difficult to observe intact root systems belowground, several techniques have been developed to screen deep- and shallow-rooting phenotypes in rice. Here, we introduce a simple and convenient method for deep- and shallow-rooting phenotyping using a unique combination of sand, soil, and plastic mesh netting. Vandana, a drought-tolerant rice variety, and Dongiin, a Korean japonica rice variety, were used to analyze root phenotypes. No significant differences in root length were observed in rice grown under irrigated conditions regardless of net position, whereas roots were significantly longer, and ratio of deep root (RDR) values were significantly higher in Vandana rice grown under semidrought conditions. In summary, this simple and useful method represents a low-cost means of phenotyping the roots of rice and other crops grown in various-sized pots and at multiple plant growth stages.
\end{abstract}

Keywords Root phenotyping method, Deep rooting, Root architecture, Drought stress tolerance

\section{Introduction}

Although rice is a common food for more than half of the

J.-H. Han $\cdot$ N.-H. Shin $\cdot$ J.-H. Moon

Department of Plant Life \& Environmental Science, HanKyong

National University, Anseong, 17579, Korea

J. H. Chin

Graduate School of Integrated Bioindustry, Sejong University, Seoul, 05006, Korea

S.-C. Yoo $(\bowtie)$

Department of Plant Life \& Environmental Science, HanKyong National University, Anseong, 17579, Korea

Institute of Ecological Phytochemistry, Hankyong National

University, Anseong, 456-749, Korea

e-mail: scyoo@hknu.ac.kr world's population, and is an especially important staple in Asia, it requires ample water for growth, and as such, water scarcity can significantly reduce rice-crop yields. Rice is particularly susceptible to drought-induced stress due to its relatively shallow rooting compared to other cereal crops (Angus et al. 1983), which limits its ability to tap into water supplies that lie below $\sim 60 \mathrm{~cm}$ in the soil horizon (Fukai and Inthapan 1988).

Deep rooting, which enhances a plants' ability to extract water from deeper soil layers, is a useful strategy for avoiding drought-induced stress (Uga et al. 2013). Plants use various strategies to overcome drought stress, including drought escape, tolerance, recovery, and avoidance (Levitt 1972; O’Toole and Chang 1979). Among these four strategies, drought avoidance is most likely associated with the mode of drought resistance with roots. The component traits of plant drought avoidance consist of deep roots with high levels of branching and penetration, a high root/shoot ratio, elasticity in leaf rolling, early stomatal closure, and high cuticular resistance (Blum et al. 1989; Samson et al. 2002; Wang and Yamauchi 2006). Because deep rooting enables plants to extract water from deeper soil layers and thus aids plants in reducing drought stress (Yoshida and Hasegawa 1982; Fukai and Cooper 1995; Uga et al. 2011), modifications in root architecture and the ability to adopt deep rooting are desirable traits in the breeding of drought-resistant rice varieties (Gowda et al. 2011; Uga et al. 2011).

Deep rooting is a complex interaction between root growth angle and maximum root length (Abe and Morita 1994; Araki et al. 2002). Shallow rooting is favorable for the uptake of nutrients, such as phosphorus, from topsoil layers, whereas deep rooting is beneficial for acquiring water from subsoil layers when plants are subjected to drought conditions (Lynch 2013). In addition, as nitrates are often transported to deeper soil layers by precipitation, deep rooting enhances deep-soil nitrate absorption (Trachsel et al. 2013). Therefore, deeprooting phenotypes should be assessed for potential use in the development of more drought-tolerant crop varieties. 
Direct observation of intact root phenotypes belowground is challenging. Several methods for root phenotyping have been designed to screen deep- and shallow-rooting phenotypes; for example, the soil-filled glass rhizotron method uses a thin, glass rhizotron within which plants are grown (Shashidhar et al. 2012). The rhizotron is filled with soil and inclined at an angle of $15^{\circ}$, and the roots of the growing plant are observed through a glass slide, from which root traits, such as rooting angle and depth, can be measured whenever necessary. However, this technique requires specialized equipment (i.e., a rhizotron) and three-dimensional (3D) root structures cannot be measured.

A second approach, known as the pinboard method, involves growing plants in a pinboard-shaped root box (Shashidhar et al. 2012), which facilitates the collection of the whole-root system with minimal physical damage. It can also be used to evaluate root-system development and for assessing plant water usage under controlled soil moisture conditions. In some cases, however, overlapping of the roots complicates determination of root maximum lengths; moreover, this method also does not permit the development of 3D root systems.

A root phenotyping method using 3D imaging software was recently developed for examining the architecture and development of roots of plants grown in gellan gum (Clark et al. 2011). This approach captures rotational $2 \mathrm{D}$ images of plants daily during seedling development, from which 3D root images are reconstructed using the custom-designed software RootReader3D (www.plantmineralnutrition.net). One advantage of this method is that it can be used to identify phenotypes in a wide variety of ways and can shorten root system capture time to within $5 \mathrm{~min}$.

A third approach is the well-known and widely used basket method, which consists of growing plants in plastic mesh baskets; this technique permits straightforward quantitative evaluations of shallow or deep rooting (Shashidhar et al. 2012), as deep rooting is determined by the position at which the adventitious roots pass through the bottom mesh of the hemispherical mesh basket. Kato et al. (2006) evaluated the phenotype of deep rooting from the frequency of relatively high root growth angles $\left(50^{\circ}\right.$ to $90^{\circ}$ with respect to the horizontal) in rice. The basket method enables researchers to easily count roots that emerge from each part of the basket, and thus, it can be applied to numerous crop types, such as wheat (Oyanagi et al. 1993). However, finding suitably sized baskets can be difficult, depending on the plant under investigation, and roots may be damaged when plants are removed from the soil.

Here, we introduce an alternative approach to root phenotyping that we called the plastic net method, which simply consists of a layer of plastic mesh netting set between soil above and sand below. This method is useful for distinguishing deep and shallow rooting, as well as root lengths, of plants grown in pots of various sizes. Moreover, it also allows for observation of changes in the root lengths and architecture of plants grown under various environmental conditions simply by altering the position of the netting to accord with specific experimental purposes.

\section{Materials and methods}

Plant materials and growth conditions

To develop the plastic net method for use in root phenotyping applications, seeds of two rice cultivars-Dongin, an elite Korean japonica variety, and Vandana, a drought-tolerant Indian upland hybrid of a tropical japonica/aus cross (Venuprasad et al. 2007) were sterilized in $2 \%$ (v/v) sodium hypochlorite for $10 \mathrm{~min}$, rinsed twice thoroughly with deionized water for 10 min, and germinated for $3 \mathrm{~d}$ at $30^{\circ} \mathrm{C}$ in darkness. Autoclaved beach sand $\left(121^{\circ} \mathrm{C}\right.$ under 20 psi for at least $\left.30 \mathrm{~min}\right)$ was poured in the bottom of $12.5 \mathrm{~cm} \times 12.5 \mathrm{~cm} \times 11.5 \mathrm{~cm}$ pots and covered with plastic mesh netting, which was in turn covered by autoclaved soil, until the pots were filled completely. Net position varied horizontally, with nets placed $1.5 \mathrm{~cm}, 3.5 \mathrm{~cm}$, and $6 \mathrm{~cm}$ below the soil surface. Germinated rice seeds were sown in each of the pots, and the pots were kept in a growth chamber $\left(30^{\circ} \mathrm{C} / 25^{\circ} \mathrm{C}\right.$, day/night $)$ with a 12 -h photoperiod for 4 weeks.

Irrigated and semi-drought conditions

Rice seedlings were grown under two different growth conditions simulating irrigated and semi-drought conditions. For irrigated conditions, the seeds were germinated in the dark with no irrigation, following which the germinated shoots were provided with enough water to cover half of the pot for a period of 1 week, then enough water was supplied to submerge the contents of the pot over the rest of the growth period. To simulate semi-drought conditions, seedlings were provided with $70 \%$ of the field-capacity water volume for the entire growth period.

Root phenotyping

Four-week-old rice plants were dried for 2-3 days and then removed from the pots. After carefully washing excess sand from the roots, the lengths of the longest three major shoots 
extending from the bottom of the soil were measured. The ratio of deep rooting (RDR) was defined as the number of roots that penetrated the inner part of the designated circle of the mesh netting divided by the total number of roots that penetrated the soil layer:

$\mathrm{RDR}=$ the number of roots in the inner circle / the number of total roots

Inner circles were defined as the location of the $50^{\circ}, 60^{\circ}$, and $70^{\circ}$ angles from the horizontal centered on the stem of the rice plant, in the upper, middle, and lower positions of the netting, respectively (Fig. 1). The mesh size of the plastic netting was sufficiently large enough $(2 \mathrm{~mm})$ to negate possible mesh interference with root emergence. Coordinates were marked to measure the root distributions.

\section{Results and Discussion}

Process of plastic net method for root phenotyping

Several methods have been developed to facilitate observation of root phenotypes belowground, and although these methods are well designed and useful for observing root architecture, most require specialized equipment or are otherwise limited.
A

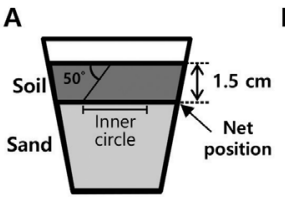

B

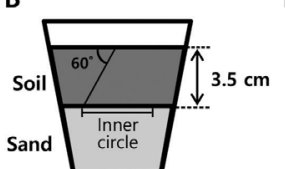

C

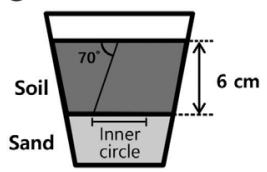

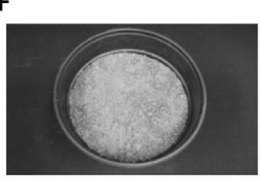

G

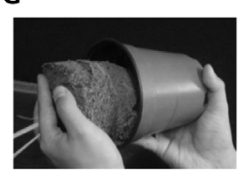

E

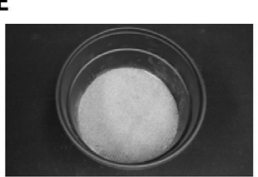

H
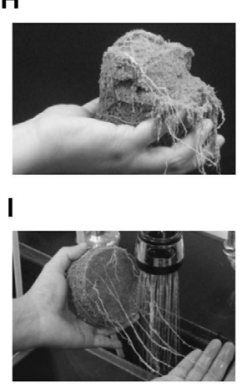

Fig. 1 Schematic diagram of the net position and the plastic net method process. The plastic mesh netting was positioned $1.5 \mathrm{~cm}$ (A), $3.5 \mathrm{~cm}(\mathrm{~B})$, and $6 \mathrm{~cm}$ (C) below the surface layer of soil in the pots. Beach sand was poured into the bottom of each pot (D), plastic netting was put on top of the sand (E), and then pots were filled with soil to the neck boundary of the pot (F). After 4 weeks, plants were carefully removed from the pots $(\mathrm{G}$, $\mathrm{H}$ ), and the sand was rinsed away. The angles of $50^{\circ}$ (A), $60^{\circ}$ (B), and $70^{\circ}(\mathrm{C})$ were defined from the horizontal, centered on the stem of the rice plant, in the upper, middle, and lower positions of the nets, respectively
Thus, we developed the plastic net method, a very simple and convenient technique that requires no special tools and minimizes physical damage during root phenotyping. In this method, plants are grown in pots filled with sand in the bottom and soil on top, with plastic mesh netting set between these two layers; the netting allows for determination of root emergence, enabling estimations of whole-root-system distribution. To select the appropriate net position for root phenotyping of plants grown under various water supply conditions, three different net positions were used, consisting of high $(1.5 \mathrm{~cm}$ below the surface), middle ( $3.5 \mathrm{~cm}$ below the surface), and low (6 $\mathrm{cm}$ below the surface) (Fig. $1 \mathrm{~A}-\mathrm{C}$ ). Thus, the root growth angles of roots growing within the inner circle would exceed $50^{\circ}, 60^{\circ}$, and $70^{\circ}$ in the high, middle, and low net positions, respectively.

To prepare cultivating soil in pots, seedling paper was first placed on the bottom of the pot to prevent fine-scale sand from leaking, then sand was poured on top of the paper, with the plastic mesh net placed on top of the sand (Fig. 1D). The top layer of sand must be smooth and evenly flat prior to net placement in order to maximize root phenotyping precision (Fig. 1E). After net placement, soil was poured on top of the net and the sand, until the pot was filled to the neck boundary (Fig. 1F). Germinated rice seeds were sown in the center of the pot and plants were allowed to grow for 4 weeks., Plants were dried for 2-3 days before removal from pots, to facilitate safe handling; plants were removed when the soil had hardened (Fig. 1G), and sand in the lower section of the pots was carefully rinsed away with running water (Fig. 1H, I). After allowing the roots to dry slightly, we marked the position of the roots on paper and prepared the plants for imaging.

\section{Root phenotyping in irrigated conditions}

Plants typically produce more roots and develop deeper root systems under water-deficient conditions to access water from deeper soil layers, whereas roots develop more slowly under irrigated conditions (Yoshida and Hasegawa 1982). In order to examine root lengths and distribution using the plastic net method, the rice varieties Dongjin and Vandana were grown under two different moisture regimes, irrigated and semi-drought. To determine the optimal net position for observing root phenotype, including root length and the RDR, three different net positions were used, as described above. Four-week-old Dongjin and Vandana seedlings grown under irrigated conditions were removed from pots, and after rinsing the roots with running water to free them from sand, the root phenotype was observed. First, root lengths of the Dongin and Vandana varieties were measured, consisting of the 
length from the bottom of the soil layer to the end of the three longest roots for each plant. The roots of Vandana rice were slightly longer in the high and low net positions (Fig. 1A, C, D and F), but no significant difference was observed between the two varieties in pots containing middle-positioned nets (Fig. 1B, E), suggesting that the root lengths did not differ substantially between Dongin and Vandana. To test whether Dongjin and Vandana exhibited deep rooting or shallow rooting under irrigated conditions, we estimated the RDRs for all net positions; no significant differences between Dongjin and Vandana were detected for the high net position (Fig. 1J), whereas RDR was significantly lower in Vandana rice than in Dongjin rice for the middle and low net positions (Fig. 1K, L), indicating that Vandana plants do not invest in extensive root growth when water is in sufficient supply.

\section{Root phenotyping in semi-drought conditions}

One strategy adopted by some plants for avoiding drought stress is to modify root architecture and distribution from shallow rooting to deep rooting in order to acquire water from deeper soil layers (Gowda et al. 2011). To establish the appropriate net position for root phenotyping of plants grown under water-limited conditions, we grew Dongin and Vandana seedlings under semi-drought conditions. Four-week-old plants were used to observe root phenotypes, including root lengths and RDR values. Interestingly, roots were markedly longer in Vandana rice than in Dongjin rice for all three net positions (Fig. 3A-F), a pattern that differed from that of rice grown under irrigated conditions (Fig. 2); this suggests that Vandana is sensitive to water deficiency and promoted root growth in response to insufficient easily available water. To test whether Dongjin and Vandana adopt deep rooting or shallow rooting under semi-drought conditions, we estimated the RDR values using the same procedures as described above. Not surprisingly, RDR values were significantly higher in Vandana for both high (Fig. 3G, J) and middle (Fig. 3H, K) net positions, and slightly higher for the low net position, although this difference was not statistically significant (Fig. 3I, L). Vandana plants thus appear to modify their root architecture and distribution from shallow rooting to deep rooting in order to acquire water from deeper soil layers when subjected to water-restricted conditions, most likely as an adaptive drought-tolerance mechanism. Thus, given the significant increases in both root lengths and RDR values displayed by Vandana rice grown in pots containing both high and middle net positions, we concluded that either of the two positions would be suitable for root phenotype observation under semi-drought conditions; however, great care must be taken so as not to disrupt the thin

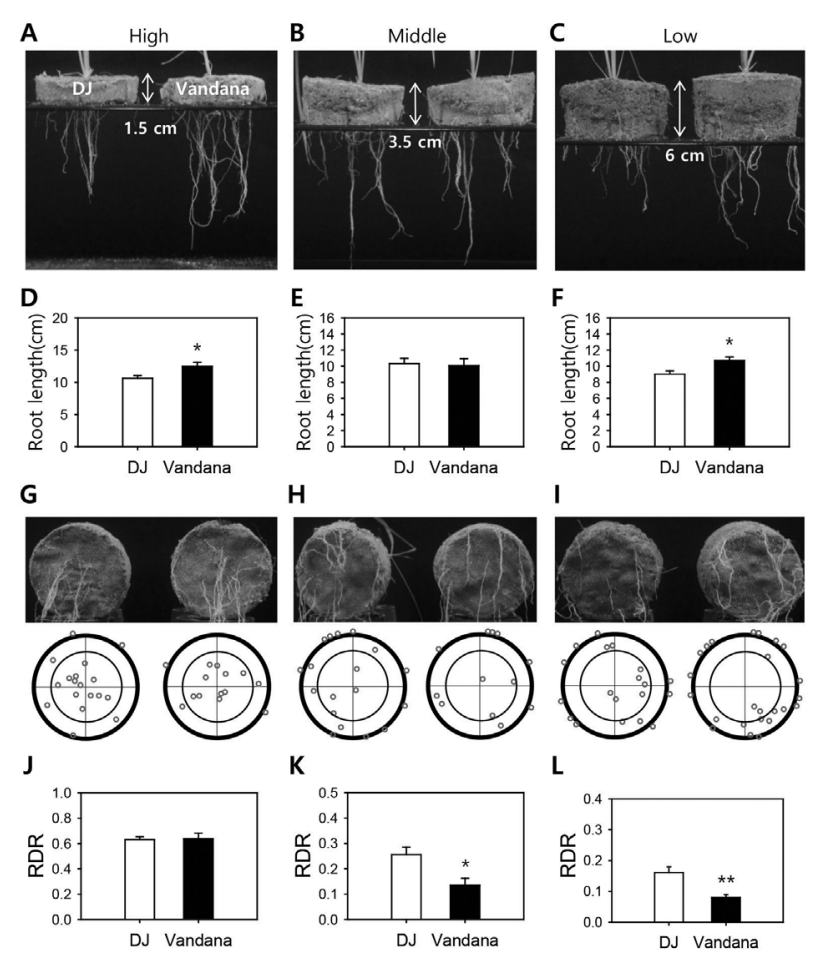

Fig. 2 Root phenotypes of Dongjin (DJ) and Vandana rice grown under irrigated conditions. Four-week old Dongjin and Vandana rice plants grown in irrigated soils were removed from pots containing three different net positions: high (A, D, G, and $\mathrm{J})$, middle (B, E, H, and $\mathrm{K}$ ), and low (C, F, I, and L). Photos show front views $(A, B$, and $C)$ and bottom views $(\mathrm{G}, \mathrm{H}$, and I) following removal of the sand layer. Root lengths (D, E, and F) and RDRs (J, K, and L) of both varieties were measured; ${ }^{* *} p<0.01,{ }^{*} p<0.05$. Root positions were marked and separated into two categories, "within the inner circle" and "outside of the inner circle" (bottom panel of G, H, and I). Dashed lines indicate the inner circle, and the root position on the outside (outside of outer circle) represents roots that emerged from the lateral part of the soils. RDR was defined as the number of roots that penetrated the inner part of the designated circle of mesh net divided by the total number of roots that penetrated the soil layer. Irrigated conditions were simulated by submerging plants in water beginning 1 week after germination

soil layer when handling plants grown in pots containing a high net position. As such, we recommend that the middle net position be used for root phenotyping in semi-drought conditions.

Case study of root phenotyping using the plastic net method with a drought-tolerant rice variety grown in semi-drought conditions

Based on our results, we concluded that the middle net position is suitable for root phenotyping in semi-drought conditions. Thus, we conducted a case study of root phenotyping using drought-tolerant and control rice varieties. We grew Nona Bokra (a salt and drought-tolerant rice variety) (Almeida et al. 

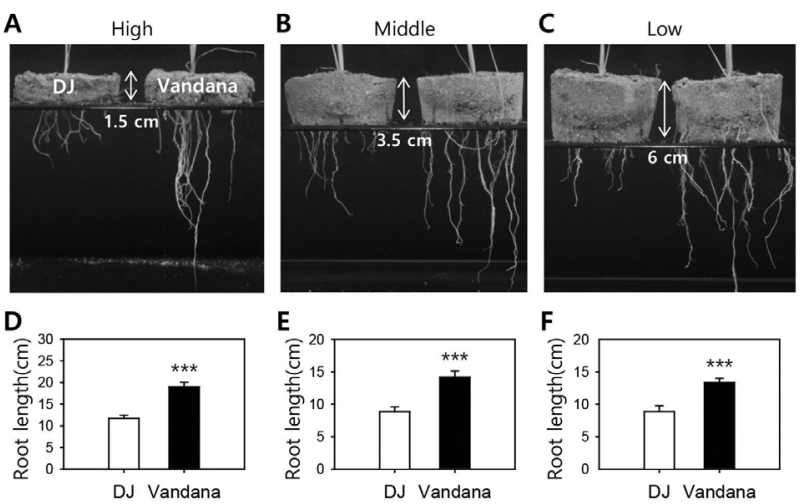

G

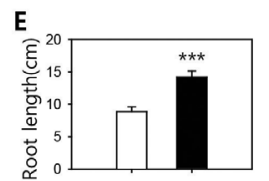

DJ Vandana

$\mathrm{H}$
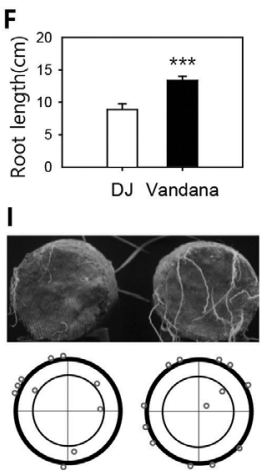

L

K

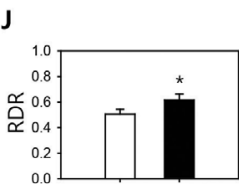

DJ Vandana

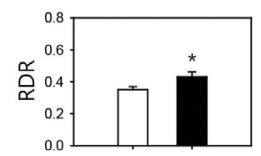

DJ Vandana

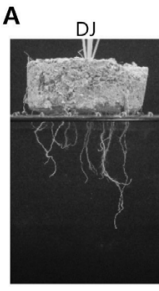

B
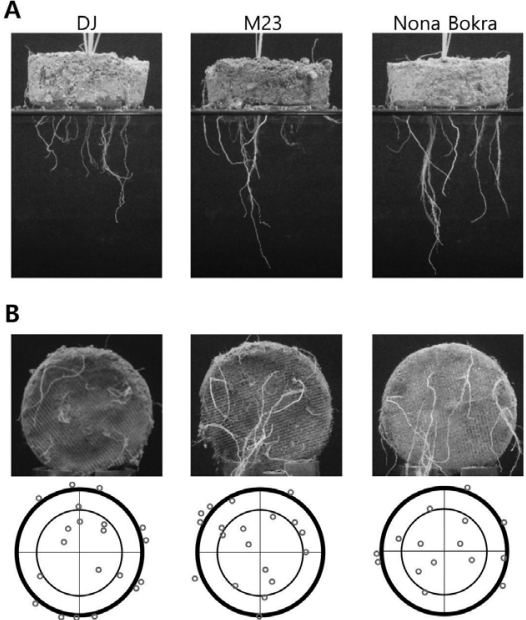

C

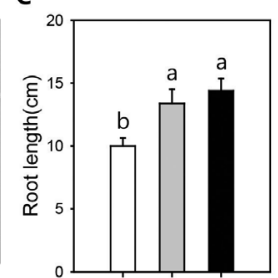

D

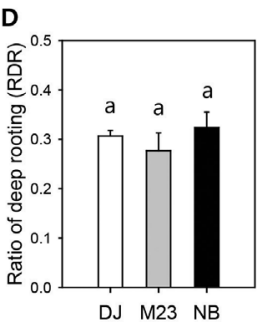

Fig. 4 Root phenotyping of rice plants grown under semi-drought conditions. Four-week old Nona Bokra, a drought-tolerant variety, and two control varieties, Dongjin and Milyang23, grown under semi-drought conditions were used for root phenotyping. Photos show front views (A) and bottom views (B) following removal of the sand layer. Root positions were marked and separated into two categories, "within the inner circle" and "outside of the inner circle" (lower panel in B). Root lengths (C) and RDRs (D) of the three varieties were measured. Letters above the bars represent statistical significance $(\mathrm{P}<0.01)$ as determined by Duncan's multiple range tests. $\mathrm{M} 23=$ Milyang23; $\mathrm{NB}=$ Nona Bokra

Fig. 3 Root phenotypes of Dongjin (DJ) and Vandana rice grown under semi-drought conditions. Four-week old Dongjin and Vandana plants grown under semi-drought conditions were removed from pots containing three different net positions: high $(A, D, G$, and $\mathrm{J})$, middle (B, E, H, and $\mathrm{K}$ ) and low (C, F, I, and L). Photos show front views $(A, B$, and $C$ ) and bottom views $(G, H$ and I) following removal of the sand layer. Root lengths (D, E, and F) and RDRs (J, K, and L) of both varieties were measured; $* * * \mathrm{p}<0.001, * \mathrm{p}<0.05$. Root positions were marked and separated into two categories, "within the inner circle" and "outside of the inner circle" (bottom panels of $\mathrm{G}, \mathrm{H}$, and I). The dashed lines and RDR are the same as in Fig. 2. Semi-drought conditions were simulated by growing seedlings under a water regime equal to $70 \%$ of field-capacity water volume over the entire growth period

2016; Biswas et al. 2002) and Dongjin and Milyang23 (non-drought-tolerant japonica and indica rice varieties, respectively) under semi-drought conditions in pots containing nets in the middle position. This experiment was carried out using the same protocols as the experiment described above. Phenotypic analysis revealed that although Nona Bokra and Milyang23 grew longer roots than Dongjin (Fig. 4A, C), there were no significant differences in RDR values among the three varieties (Fig. 4B, D). Interestingly, neither root length nor RDR were significantly higher in Nona Bokra rice than in the control indica variety Milyang23, despite Nona Bokra being known as a drought-tolerant variety. This suggests that Nona Bokra does not promote root length to any significant degree, nor does it adopt a deep-rooting strategy under waterlimited conditions, which differs from the pattern displayed

by Vandana grown under semi-drought conditions. Thus, it would appear that Nona Bokra relies on a different strategy for overcoming water-deficit stress; further research is thus needed to elucidate the drought-tolerance mechanisms used by this variety.

In this study, we found that the rice variety Vandana exhibited a very unique pattern of response to water-supply conditions; under irrigated conditions, Vandana plants adopt a shallow rooting pattern with a low root angle from the horizontal, whereas the root architecture of Vandana plants grown under semi-drought conditions changed from shallow to deep rooting with a high root angle to access water from deeper soil layers, a common strategy among drought-tolerant plants in response to drought stress.

The plastic net method is an effective tool for root phenotyping, and can be used for determination of root length, architecture, and distribution. Although the underlying principle of this method is quite similar to that of the basket method (Shashidhar et al. 2012), it does not have the same limitations, such as finding baskets that match the various pot sizes and the potential for root damage during plant removal from the soil. As such, the plastic net method developed in this study has several advantages over the basket method: for one, no specialized equipment or materials other than simple plastic mesh netting are required; for another, this technique minimizes the potential of physical 
damage to the roots via the use of sand instead of soil in the bottom of the pots; and lastly, the net method can be applied to various kinds of pot experiments at a relatively low cost. Furthermore, although here we restricted testing of this method to rice systems, this approach can be applied to root phenotyping of many other crop systems in addition to rice.

\section{Acknowledgements}

This work was supported by a research grant from Hankyong National University in the year of 2014.

\section{References}

Abe J, Morita S (1994) Growth direction of nodal roots in rice: its variation and contribution to root system formation. Plant Soil, 165(2):333-337

Almeida DM, Almadanim MC, Lourenço T, Abreu IA, Saibo NJ, Oliveira MM (2016) Screening for Abiotic Stress Tolerance in Rice: Salt, Cold, and Drought. Environmental Responses in Plants: Methods and Protocols, 155-182

Araki H, Morita S, Tatsumi J, Iijima M(2002) Physiol-morphological analysis on axile root growth in upland rice. Plant Prod Sci, 5(4):286-293

Angus JF, Hasegawa S, Hsiao TC, Liboon SP, Zandstra HG (1983) The water balance of post-monsoonal dryland crops. J Agric Sci, 101(03):699-710

Biswas J, Chowdhury B, Bhattacharya A, Mandal AB (2002) In vitro screening for increased drought tolerance in rice. In Vitro Cellular \& Developmental Biology-Plant, 38(5):525-530

Blum A, Mayer J, Golan G (1989) Agronomic and physiological assessments of genotypic variation for drought resistance in sorghum. Crop Pasture Sci, 40(1):49-61

Clark RT, MacCurdy RB, Jung JK, Shaff JE, McCouch SR (2011) Three-dimensional root phenotyping with a novel imaging and software platform. Plant Physiol, 156(2):455-465

Fukai S, Cooper M(1995) Development of drought-resistant cultivars using physiomorphological traits in rice. Field Crops Res, 40(2):67-86

Fukai S, Inthapan P (1988) Growth and yield of rice cultivars under sprinkler irrigation in south-eastern Queensland. 3. Water extraction and plant water relations dash comparison with maize and grain sorghum. Anim Prod Sci, 28(2):249-252

Gowda VR, Henry A, Yamauchi A, Shashidhar HE, Serraj R (2011) Root biology and genetic improvement for drought avoidance in rice. Field Crops Res, 122(1):1-13

Kato Y, Abe J, Kamoshita A, Yamagishi J (2006) Genotypic variation in root growth angle in rice (Oryza sativa L.) and its association with deep root development in upland fields with different water regimes. Plant Soil, 287(1-2):117-129

Levitt J, Levitt J (1972) Responses of plants to environmental stresses. New York: Academic press

Lynch JP (2013) Steep, cheap and deep: an ideotype to optimize water and $\mathrm{N}$ acquisition by maize root systems. Ann Bot, 112(2):347-357

O'Toole JC (1979) Drought resistance in cereals: rice, a case study Oyanagi A, Nakamoto T, Wada M (1993) Relationship between root growth angle of seedlings and vertical distribution of roots in the field in wheat cultivars. Jpn. J. Crop. Sci. 62:565-570

Samson BK, Hasan M, Wade LJ (2002) Penetration of hardpans by rice lines in the rainfed lowlands. Field Crops Res, 76(2):175-188

Shashidhar HE (2012) Methodologies for root drought studies in rice. (eds) Int Rice Res Inst

Trachsel S, Kaeppler SM, Brown KM, Lynch JP (2013) Maize root growth angles become steeper under low $\mathrm{N}$ conditions. Field Crops Res, 140:18-31

Uga Y, Okuno K, Yano M (2011) Drol, a major QTL involved in deep rooting of rice under upland field conditions. J Exp Bot, 62(8):2485-2494

Uga Y, Sugimoto K, Ogawa S, Rane J, Ishitani M, Hara N, Inoue H (2013) Control of root system architecture by DEEPER ROOTING 1 increases rice yield under drought conditions. Nature Genet, 45(9):1097-1102

Uga Y, Yamamoto E, Kanno N, Kawai S, Mizubayashi T, Fukuoka $\mathrm{S}$ (2013) A major QTL controlling deep rooting on rice chromosome 4. Sci Rep, 3:3040

Venuprasad R, Lafitte HR, Atlin GN (2007) Response to direct selection for grain yield under drought stress in rice. Crop Sci, 47(1):285-293

Wang H, Yamauchi A (2006) Growth and function of roots under abiotic stress in soils. In B. Huang (eds) Plant-Environment Interactions, Third Edition (pp. 271-319). CRC Press

Yoshida S, Hasegawa S (1982) The rice root system: its development and function. Drought resistance in crops with emphasis on rice, Los Banos, Philippines: Int Rice Res Inst, 97-114 